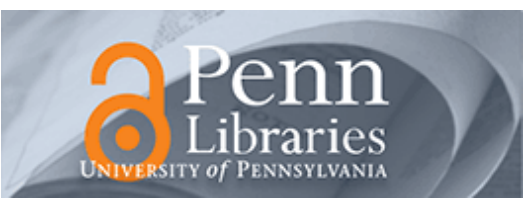

University of Pennsylvania

ScholarlyCommons

December 1996

\title{
A simplified model based supercritical power plant controller
}

\author{
Wataro Shinohara \\ Toshiba Corporation \\ Daniel E. Koditschek \\ University of Pennsylvania, kod@seas.upenn.edu
}

Follow this and additional works at: https://repository.upenn.edu/ese_papers

\section{Recommended Citation}

Wataro Shinohara and Daniel E. Koditschek, "A simplified model based supercritical power plant controller", . December 1996.

Copyright 1996 IEEE. Reprinted from Proceedings of the 35th IEEE Conference on Decision and Control, Volume 4, 1996, pages 4486-4491.

This material is posted here with permission of the IEEE. Such permission of the IEEE does not in any way imply IEEE endorsement of any of the University of Pennsylvania's products or services. Internal or personal use of this material is permitted. However, permission to reprint/republish this material for advertising or promotional purposes or for creating new collective works for resale or redistribution must be obtained from the IEEE by writing to pubs-permissions@ieee.org. By choosing to view this document, you agree to all provisions of the copyright laws protecting it.

NOTE: At the time of publication, author Daniel Koditschek was affiliated with the University of Michigan. Currently, he is a faculty member in the Department of Electrical and Systems Engineering at the University of Pennsylvania.

This paper is posted at ScholarlyCommons. https://repository.upenn.edu/ese_papers/375

For more information, please contact repository@pobox.upenn.edu. 


\title{
A simplified model based supercritical power plant controller
}

\author{
Abstract \\ We present a simplified state-space model of a once-through supercritical boiler turbine power plant. This \\ phenomenological model has been developed from a greatly simplified application of the first principles \\ of physical laws. When we fit our model to a far more complex and physically accurate simulation model \\ commissioned by EPRI for operator training, we find that the input-output responses are surprisingly \\ close. \\ Encouraged by this initial success, we describe some initial steps toward a design method for \\ supercritical boiler control suggested by the geometric structure arising from the simplified model. \\ Preliminary simulation results suggest that this approach may offer a closed loop response considerably \\ improved relative to that achieved by the linear controllers presently in place in typical industrial settings. \\ Comments \\ Copyright 1996 IEEE. Reprinted from Proceedings of the 35th IEEE Conference on Decision and Control, \\ Volume 4, 1996, pages 4486-4491. \\ This material is posted here with permission of the IEEE. Such permission of the IEEE does not in any way \\ imply IEEE endorsement of any of the University of Pennsylvania's products or services. Internal or \\ personal use of this material is permitted. However, permission to reprint/republish this material for \\ advertising or promotional purposes or for creating new collective works for resale or redistribution must \\ be obtained from the IEEE by writing to pubs-permissions@ieee.org. By choosing to view this document, \\ you agree to all provisions of the copyright laws protecting it. \\ NOTE: At the time of publication, author Daniel Koditschek was affiliated with the University of Michigan. \\ Currently, he is a faculty member in the Department of Electrical and Systems Engineering at the \\ University of Pennsylvania.
}




\section{A Simplified Model Based Supercritical Power Plant Controller}

\author{
Wataro Shinohara ${ }^{1}$
}

\section{Abstract}

We present a simplified state-space model of a oncethrough supercritical boiler turbine power plant. This pheonomenological model has been developed from a greatly simplified application of first principles physical laws borrowing from (and further simplifying) the assumptions of some previous authors. When we fit our model to a far more complex and physically accurate simulation model commissioned by EPRI for operator training, we find that the input-output responses are surprisingly close.

Encouraged by this initial success, we describe some initial steps toward a design method for supercritical boiler control suggested by the geometric structure arising from the simplified model. Very preliminary simulation results suggest that this approach may offer a closed loop response considerably improved relative to that achieved by the linear controllers presently in place in typical industrial settings.

\section{Introduction}

Fossil fueled power plants convert chemical energy to electrical energy through the coordinated exchange of various intermediate forms of energy. A number of distinct physical processes - fuel combustion in the furnace, heat transfer from flue gas to working fluid (water/stearn) through the boiler wall, mechanical movement of turbine blades resulting from steam enthalpy drop - are all involved in the complete con cycle. The dynamic reponse of a power station is determined primarily by its "slowest" physics - heat exchange between the furnace and the working fluid. In this paper, we propose a simple model of heat exchange for a supercritical boiler turbine power station compared with a far more rigorously built simulator developed

\footnotetext{
${ }^{1}$ Research and Development Center, Toshiba Corporation, 4-1 Ukishima-cho, Kawasaki-ku, Kawasaki 210 JAPAN

${ }^{2}$ Department of Electrical Engineering and Computer Science, University of Michigan, Ann Arbor, MI 48109-2110

${ }^{3}$ This work was supported in part by the Electric Power Research Institute under Grant RP 8030-17. The authors would like to acknowledge the advice and helpful criticism of Prof. E. Lady and Prof. R. Smoak.
}

\author{
Daniel E. Koditschek ${ }^{2}$
}

for EPRI[6] for operator training. We next propose a controller design approach which utilizes the geometric structure of this model to improve its control performance.

There presently exist very accurate power station models designed for exhaustive testing and analysis of alternative control policies. The EPRI[6] simulator is a good example. Missing still, in our view, is a more phenomenological and higher level understanding of the large signal behavior of fossil fuel plants that might promote the design of more effective controllers integrating various levels of operation in a predictable manner. There seems to exist a small controls motivated literature for drum-type boiler/turbine plant, notably [3], and, very recently, [2]. In this work, however, we are interested exclusively in supercritical boilers wherein heat exchange takes place under pressures and temperatures well over the critical point of water. We adopt in greatly simplified form the modeling point of view introduced in [4] and expanded in [5]. We impose additional simplifying assumptions in order to obtain the simplest dynamical system that exhibits many of the salient characteristics of the physical phenomena of interest.

After briefly describing our model in the next section, we present some simulation comparisons with the EPRI simulator in Section 3 by way of justifying our claim to have captured some of the key dynamical characteristics of the physical plant in simplified form. Next, the new approach to controller design is described in Section 4 including some simulation results for the performance validation. We conclude the paper by discussing the subjects to be addressed in succeeding work.

\section{Model}

\subsection{Assumptions}

In order to derive the plant model, we first adopt many of the assumptions originally introduced in [4], such as uniform fluid property at any cross section, no gaspressure dynamics, balanced flow and so on. In the interest of still greater simplicity, we depart from the earlier work by further assuming

- there are only two sections in the working fluid path - the furnace and the superheater 
- work by a set of the turbines, that is, high/intermediate/low pressure turbines, is represented as the work of a single turbine unit.

- due to the turbine assumption, the effect of the reheater section is taken into account in the superheater section

- steam pressure dynamics is ignored $(\dot{P}=0)$

- the working fluid displays constant mass flow rate in the superheater but variable mass flow rate through the furnace section

The first of these new assumptions may be easily relaxed to scale our model back up to the previous literature. As for the turbines, each turbine works almost identically from the viewpoint of the "enthalpy drop" through the unit, and their coordinated effect seems plausibly represented by a single turbine's. This assumption forces us to treat the superheater and the reheater as a single compartment. These assumptions could also easily be relaxed, if the model were scaled up, and the turbines and the reheater evaluated separately. The last assumption lumps all the compressibility of the working fluid into the furnace section, yoking the density of the working fluid on either side of this compartment to the more directly controlled flow rates. ${ }^{1}$. For detailed discussion of the assumptions, please see [1].

\subsection{Basic model description}

As mentioned, we are most centrally interested in the mechanism of heat transfer from combustion to steam generation - the dominant dynamical feature of the power generation process.

Fig. 1 presents a simple block diagram of our plant model labelled with the principal variable names. In this formulation, water flow rate from economizer, $w_{f w}$, throttle valve opening, $u_{v}$, and heat transfer from wall, $Q_{w f}, Q_{w s}$ (both assumed to be proportional to combustion heat $Q_{c}$, are regarded as control inputs, and generated power, $W$, is the output. Highly compressed water from the economizer, $w_{f w}$, flows through the furnace and the superheater receiving heat transferred from the wall in each section, $Q_{w f}, Q_{w s}$, and changes to steam all in a supercritical state (i.e., without any two phase phenomena). Superheated supercritical steam is directed into the turbine via throttle valve(s) which control steam flow rate, $w_{f s}$. The latter is directly proportional to the power generated by the turbine, $W$.

The derived system is described in the state-space form as follows.

\footnotetext{
${ }^{1}$ Masada [5] considered the compressibility effect in economizer and reheater sections
}

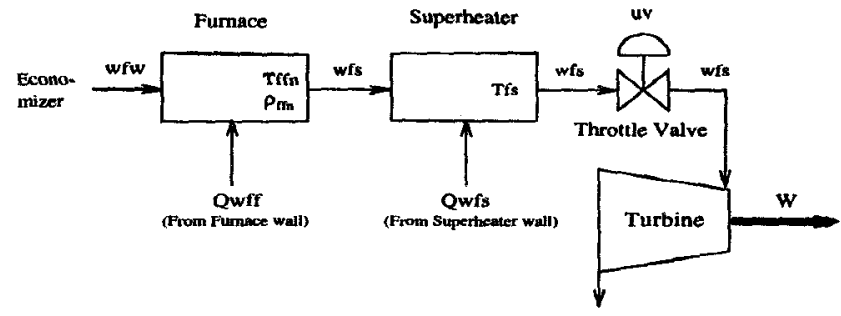

Figure 1: Simplified block diagram of a boiler-turbine power plant

$$
\begin{aligned}
\frac{d}{d t}\left(\begin{array}{c}
\rho_{f n} \\
T_{f n} \\
T_{s h}
\end{array}\right)= & \left(\begin{array}{ccc}
\frac{1}{V_{f n}} & -\frac{k_{v} P_{s h}}{V_{f n} \sqrt{T_{s h}}} & 0 \\
\frac{h_{e c}-h_{f n}}{V_{f n} \zeta_{f n}} & 0 & \frac{k_{f n} \Delta q_{c}}{V_{f n} \zeta_{f n}} \\
0 & \frac{k_{v} P_{s h}\left(h_{f n}-h_{s h}\right)}{V_{s h} \zeta_{s h} \sqrt{T_{s h}}} & \frac{k_{s h} \Delta q_{c}}{V_{s h} \zeta_{s h}}
\end{array}\right) \\
& \times\left(\begin{array}{c}
w_{f w} \\
u_{v} \\
w_{c}
\end{array}\right)
\end{aligned}
$$

$$
\begin{gathered}
W=\frac{k_{v} P_{s h}\left(h_{s h}-h_{c d}\right)}{K_{t b} \sqrt{T_{s h}}} u_{v} \\
\dot{x}=B(x) u \\
y=g(x) u
\end{gathered}
$$

\subsection{Actuator Lags}

In the actual plant configuration, we can't subsystems dynamics. The actuator/auxiliary subsystems in the plant, such as feedwater pump, are thought to be reasonably approximated as first-order lag systems. Then the total system becomes as follows.

$$
\begin{aligned}
\dot{x_{1}} & =-\Lambda\left(x_{1}-u\right) \\
\dot{x_{2}} & =B\left(x_{2}\right) x_{1} \\
y & =g\left(x_{2}\right) x_{1}
\end{aligned}
$$

where $u$ denotes the control demand we design other than the actual inputs in (4).

\section{Validation Comparison with EPRI Simulator}

Our simplified model has shown a modest ability of emulating the corresponding responses, especially $1 / \mathrm{O}$ resonses of EPRI simulator. Fig. 2 and 3 show the comparison results of generated power output and fuel flow input respectively. 


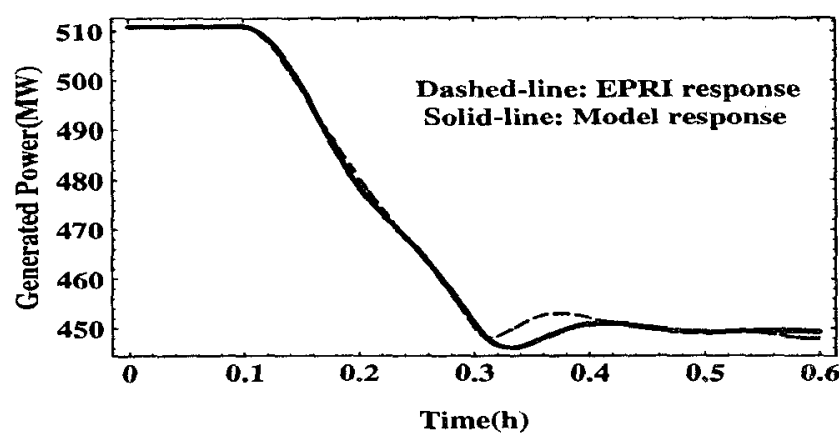

Figure 2: MW response comparison of simplified model and EPRI simulator

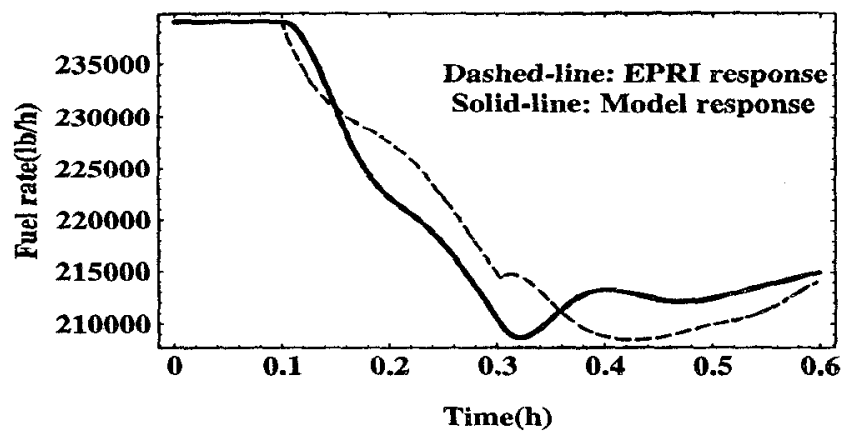

Figure 3: Fuel flow response comparison of simplified model and EPRI simulator

\section{Nonlinear Controller Design}

\subsection{Intuitive Control Strategy}

There are some requirements for the load demand following control. The most important condition is that the control action should not only make the system track the demand, but also keep some system property values such as the temperature and pressure of the steam at boiler outlet(throttle valve) within a specified range of values during its transient process. To make a rough feasibility study on such ideal control, let us imagine a crude boiler/turbine model which has 3 inputs, feedwater flow, fuel input, and throttle valve opening, together with 2 outputs, temperature and pressure of the steam at the boiler outlet. For swift tracking to load demand, we could adjust the throttle valve opening to control the steam mass flow rate into the turbine, which directly affects the generated power, as in the case of the 'boiler-following control' scheme. In case of increasing power, this overly quick opening of the valve should lead to sudden drop of pressure and internal enegy of the boiler side at the valve. To reduce the fluctuations of the properties at the valve, we need to increase the feedwater flow to keep the pressure and put more fuel into a combustion chamber to compensate the lost enegy, that is, maintain the desired temperature. So as far as we could match the feedwater flow rate with the steam flow rate at the valve, and promptly heat up the fluid to provide as much energy as needed to increase generated power to follow the demand, the temperature and pressure at the valve would never be affected. Those in the boiler control community will recognize this intuition as reflecting the same philosophy as the so-called 'boiler-following control' approach. However, away from the small signal linear domain, the traditional boiler following control typically fails to compensate adequately because it has no basis for estimating the amount of energy needed to replace the energy lost. Our proposed nonlinear controller is designed to provide this information in a form suggested by the geometric structure of the plant.

\subsection{Preliminary Setup}

4.2.1 The Plant Model: For the present we take the state space to be the vector space, $\mathcal{X}:=\mathbb{R}^{3}$, thus, the possible vector fields are defined on $T \mathcal{X} \approx \mathbb{R}^{6}$. Similarly, we take the space of control inputs to be the vector space $\mathcal{U}:=\mathbb{R}^{3}$.

The control system takes the form

$$
\begin{aligned}
& \dot{x}=B(x) u \\
& y=g(x) u,
\end{aligned}
$$

where $B$ has full rank except on a singular surface, $\mathcal{S} \subset \mathcal{X}$ of dimension two, and $g$ has rank one, always. It turns out for our plant that $g$ has a null space that has only a trivial intersection with the null space of $B$, that means we could use an null-input of $B$ to manipulate the system output $y$.

4.2.2 Notation: In the interest of simplicity, we define $\mathcal{E}:=\mathcal{X} \times \mathcal{U}$ to be the ("trivial") input bundle over the state space. Similarly, denote by $\mathcal{F}:=\mathcal{X} \times$ $\mathcal{Y}$ the ("trivial") output bundle over the state space. Hereafter, let us allow to identify $B$ as an bundle map, $B: \mathcal{E} \rightarrow T \mathcal{X}$.

As we have mentioned, $B$ drops rank, as measured by the determinant $\sigma(x):=\operatorname{det} B(x)$, on a surface

$$
\mathcal{S}:=\{x \in \mathcal{X}: \sigma \equiv 0\}
$$

of dimension two in $\mathcal{X}$ over which there is a "missing" direction of control

$$
\mathcal{K}:=\operatorname{Ker} B^{\mathrm{T}} \subset T \mathcal{X},
$$

comprising the "orthogonal complement" of $B$ over $\mathcal{S}$, and a "useless" direction of effort,

$$
\mathcal{N}:=\operatorname{Ker} B \subset \mathcal{E},
$$

comprising the "null space" of $B$ over $\mathcal{S}$. It must be emphasized that $\mathcal{K}$ coincides with the zero section of $T \mathcal{X}$, and $\mathcal{N}$ coincides with the zero section of $\mathcal{E}$ except over $\mathcal{S}$, denoted $\mathcal{K}_{\mathcal{S}}, \mathcal{N}_{\mathcal{S}}$, where they both have dimension one. 
4.2.3 Projections: In general, the projection of a bundle onto its base space will be denoted by $\pi$ -. for example, $\pi_{\mathcal{E}}$ maps points of $\mathcal{E},(x, u)$, to $x ; \pi_{T \mathcal{X}}$ maps points of $\mathcal{T X},(x, \dot{x})$, to $x$; and so on. In contrast, projections into the fiber will be denoted by $\Pi$. For example, given any non-zero $e \in \mathcal{E}$,

$$
\Pi(e):=I-\frac{1}{\|e\|^{2}} e e^{T},
$$

maps vectors, $n \in \mathcal{E}$ onto the orthogonal complement of $e,-$ that is,

$$
e^{\mathrm{T}} \Pi(e) n=0
$$

\subsection{Small Cycling Control Strategy}

In this section we assume that the plant is in a steady state condition - that is a reference load trajectory, $y^{*}(t)$, must be tracked, but its excursions are small enough that there is a "nice" setpoint, $x^{*} \in \mathcal{S} \subset \mathcal{X}$ from which the tracking can be safely accomplished.

Under such conditions, we can built an ideal control input which generates a desired output $y^{*}(t)$ while keeping the states remained near in the steady state, $x^{*}$, as the sum of two control components, $u_{\phi}$ and $u_{p}$ which we call the "surface regulator" and "demand tracker," respectively.

4.3.1 The Surface Regulator $-u_{\phi}$ : The control component $u_{\phi}$ drives the system states onto the singular surface $\mathcal{S}$ and keeps them there. Specifically, $u_{\phi}$ is designed so as to be a gradient descent to the surface $\mathcal{S}$.

Let $\varphi: \mathcal{X} \rightarrow \mathbb{R}$ be nondegenerate (that is, its hessian is full rank at every critical point) and take its minimum - say $\varphi\left(x^{*}\right)=0$ - uniquely on some $x^{*} \in \mathcal{S}$. Then the control strategy

$$
u_{\varphi}:=-B^{\mathrm{T}} \operatorname{grad} \varphi
$$

applied to (6) results in a closed loop system along whose motions $\varphi$ is decreasing

$$
\dot{\varphi}=-\left\|B^{\mathrm{T}} \operatorname{grad} \varphi\right\|^{2} \text {. }
$$

The zero set of $\dot{\varphi}$ is a disjoint union $\{\dot{\varphi} \equiv 0\}=\mathcal{C}_{\varphi} \sqcup \Phi_{\mathcal{S}}$, where we denote by $\mathcal{C}_{\varphi}$ the set of critical points (extrema) of $\varphi$ not in $\mathcal{S}$, and by $\Phi_{\mathcal{S}}$, those points in $\mathcal{S}$ where $\operatorname{grad} \varphi \in \mathcal{K}$ - including $x^{*}$. By construction, any other critical point of $\varphi, x_{\epsilon} \in \mathcal{C}_{\varphi}$, has an indefinite (if it is a saddle) or negative definite (if it is a maximum) hessian, $Q \triangleq\left[D^{2} \varphi\right]\left(x_{c}\right)$, and its instability under (8) is assured by the local Lyapunov instability function defined by $x^{\mathrm{T}} Q x$. It follows from LaSalle's invariance principle [7] that all motion of the closed loop system approach $\Phi_{\mathcal{S}}$, and, indeed, they approach $x^{*}$ if it can be guaranteed that $\operatorname{grad} \varphi \notin \mathcal{K}$.
4.3.2 The Demand Tracker - $u_{p}$ : The second component $u_{p}$ is designed to control the system output to follow the demand while being so orthogonalized to the gradient descent component $u_{\phi}$ as not to hamper its surface regulation.

Now suppose that $n$ is a smooth section of $\mathcal{E}$ that takes its image in $\mathcal{N}_{\mathcal{S}}$ - that is, $n: \mathcal{X} \rightarrow \mathcal{E}$ such that $\pi_{\mathcal{E}} \circ n \equiv$ $i d_{\mathcal{X}}$ but $n \mathcal{S} \subset \mathcal{N}_{\mathcal{S}}$. This can be achieved in such a fashion that

$$
u_{p}:=\Pi\left(B^{T} \operatorname{grad} \varphi\right) n,
$$

remains well defined on the entirety of $\mathcal{E}$. For note that while the projection $\Pi\left(B^{\mathrm{T}} \operatorname{grad} \varphi\right)(7)$ is not necessarily defined over $\mathcal{S}$ (since $B^{\mathrm{T}} \operatorname{grad} \varphi$ may vanish there), the numerator

$$
\frac{1}{\left\|B^{\mathrm{T}} \operatorname{grad} \varphi\right\|^{2}} B^{\mathrm{T}} \operatorname{grad} \varphi[\operatorname{grad} \varphi]^{\mathrm{T}} B n
$$

goes to zero with third order while the denominator goes to zero with second order as $x \rightarrow \mathcal{S}$.

The control strategy,

$$
u_{\varphi p}:=u_{\varphi}+u_{p}
$$

applied to (6) results in a closed loop system that admits $\varphi$ as a Lyapunov function using the same arguments as above and we conclude that (10) drives the plant toward $x^{*}$ as well, regardless of the form of $n$.

To assure output tracking, we may use this "one degree of freedom" in $\mathcal{N}_{\mathcal{S}}$ as follows. Denote by $\hat{n}$ a smooth "unit section" of $\mathcal{E}$ - that is, $\hat{n}: \mathcal{X} \rightarrow \mathcal{E}$ such that $\pi_{\mathcal{E}} \circ \hat{n} \equiv i d_{\mathcal{X}}$ and $\|\hat{n}\| \equiv 1$. Note that the restriction $\hat{n} \mid$ $\mathcal{S}$ is unique, but $\hat{n}$ can be any smooth extrapolation to $\mathcal{X}$. Choosing a smooth extrapolant with the property

$$
g \Pi\left(B^{T} \operatorname{grad} \varphi\right) \hat{n} \neq 0
$$

when $x \notin \mathcal{S}$ guarantees that this inequality holds for all $x \in \mathcal{X}$. This is true since $\hat{n} \equiv \Pi\left(B^{\mathrm{T}} \operatorname{grad} \varphi\right) \hat{n}$ on $\mathcal{S}$ and the plant has the property that $\operatorname{Ker} g \cap \mathcal{N}=\{0\}$. Revisiting the recipe for $u_{p}$ in (9), we may now rewrite

$$
n:=\frac{y^{*}-g u_{\varphi}}{g \Pi\left(B^{\mathrm{T}} \operatorname{grad} \varphi\right) \hat{n}} \hat{n}
$$

and it follows that the response, $y$, to $u_{\varphi p}$ is exactly $y^{*}$.

\section{Simulation Study}

\subsection{Simulation Setup}

We now show some control simulation results for each model, where the main control objects are,

- Smooth load following

- SH temperature kept as near at $1000(\mathrm{~F})$ as possible 
The version of the EPRI simulator that has been made available to us does not support linking externally developed object code (versions that do so are available at considerable cost from the TRAX corporation [6]) so in this very initial period of our study we have compared the performance of an "EPRI-style" controller against strategies arising from the geometric structure (10) developed above by running both on our very simple model (6) calibrated to the "true plant" (the EPRI simulator) as discussed in [1]. The controllers are designed in Mathematica and ported to $C$ for numerical efficiency. Control simulations are performed for short demand changes from $511 \mathrm{MW}$ to $450 \mathrm{MW}$.

The actuator lags are compensated by incorporating standard feedforward/feedback compensation loops into the control configuration (6) according to the scheme

$$
\begin{aligned}
\dot{z} & =u_{d}-x_{1} \\
u & =\left(I-K_{1}\right) x_{1}+K_{1} u_{d}+K_{0} z
\end{aligned}
$$

where $u_{d}$ is the target control strategy. The closed-loop dynamics of the actuators are now $\ddot{u}=K_{1} \Lambda^{-1}\left(u_{d}-\dot{u}\right)+$ $K_{0} \Lambda^{-1}\left(u_{d}-u\right)$, and it is clear that we can force the actuator output to follow $u_{d}$ asymptotically exactly, and as quickly as the magnitude of the gains, $K_{1}, K_{2}$ permits. With high gain settings, the plant looks essentially first order (4) and should clearly favor our nonlinear scheme (10). However, in the simulation study described below, we have set these gains rather low to avoid a reliance on unrealistically high actuator bandwidth.

\subsection{Simulation Results}

We first show simulation results of the EPRI-type linear controller in Fig. 4 and 5. Next, in Fig. 6 and 6 , we display the response to the geometric controller where $u_{d}$ is set to be (10). Despite the rather modest actuator gain settings, the geometric controller achieves considerably improved load following control performance.

\section{Linear control:511MW $\Rightarrow 450 \mathrm{MW}$}
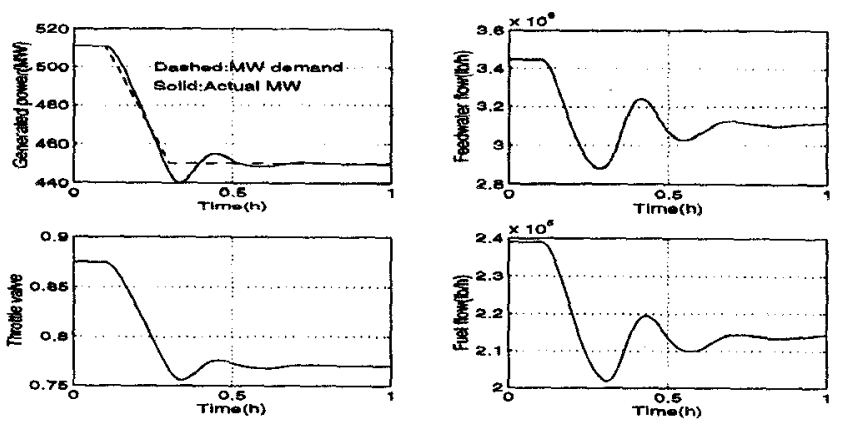

Figure 4: EPRI-type linear control - I/O responses $(511 \mathrm{MW} \rightarrow 450 \mathrm{MW})$
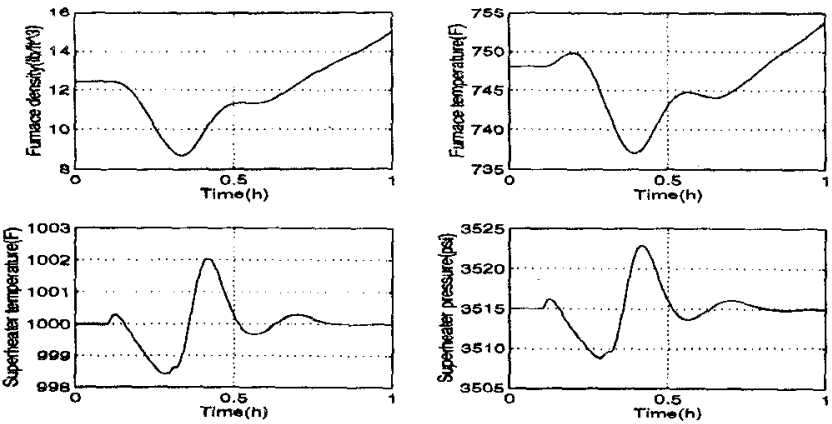

Figure 5: EPRI-type linear control - state responses $(511 \mathrm{MW} \rightarrow 450 \mathrm{MW})$

Nonlinear control:511MW $\Rightarrow 450 \mathrm{MW}$
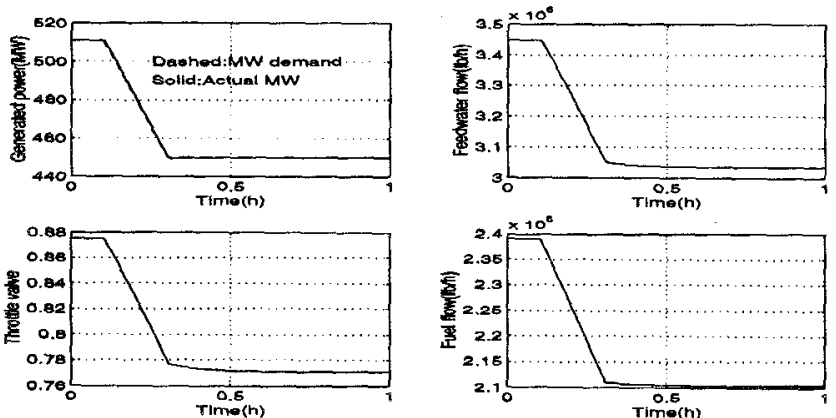

Figure 6: Nonlinear control(small lag-compensation gain) $-\mathrm{I} / \mathrm{O}$ responses $(511 \mathrm{MW} \rightarrow 450 \mathrm{MW})$
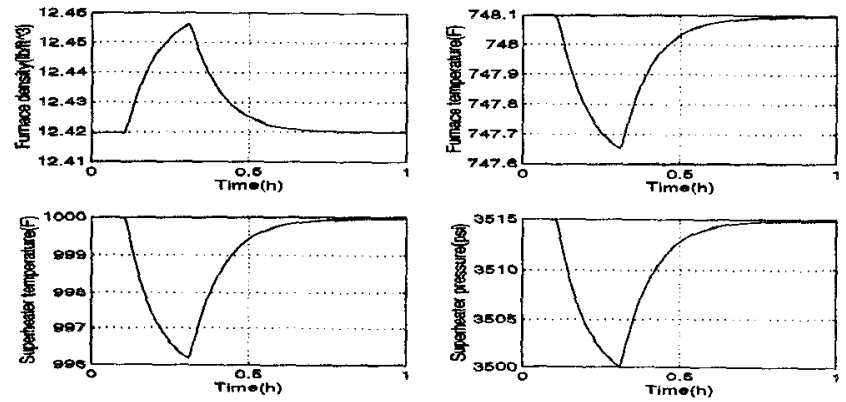

Figure 7: Nonlinear control(small lag-compensation gain) - state responses $(511 \mathrm{MW} \rightarrow 450 \mathrm{MW})$ 


\section{Conclusion - Future Work}

We have suggested the potential utility of a nonlinear controller for supercritical power plants inspired by the geometric structure of an extremely simplified but physically-oriented phenomenological model. Obviously, there remains much future work to be done before such a controller could be worthy of serious consideration in the power utility industries. We now briefly indicate some of the directions this future work must take.

Of most obvious importance, we are presently developing a much more detailed plant model including some other components such as, reheater, Intermediate/low pressure turbines, feedwater heaters and so on. This extended model will enable us to investigate in greater detail the advantages of controllers inspired by the simple model (4) and to correct the disadvantages. More specifically, several disadvantages have already become clear. Most egregiously, present simulation results reveal that the geometric controller affords inadequate regulation of the steam pressure at boiler outlet. We are presently introducing additional terms into (10) to take care of this problem.

\section{References}

[1] W.Shinohara and D.Koditschek, A simplified model for a supercritical power plant, University of Michigan, Control group reports, CGR-95-08, October 1995.

[2] G.Pellegrinetti and J.Bentsman, Nonlinear conrol oriented boiler modeling - A benchmark problem for controller design, IEEE Transactions on Control Systems Technology, Vol.4, No.1, pp.57-64, January 1996.

[3] K.J. Åström and K.Eklund, A Simplified Non-Linear Model of a Drum Boiler-Turbine Unit, Int.J.Control, Vol.16, No.1, pp.145-169, 1972

[4] J.Adams,D.R.Clark,J.R.Louis and J.P.Spanbauer, Mathematical model of once-through boiler dynamics, IEEE Transactions on Power Systems, Vol.84, pp.146-156, February 1965.

[5] G.Y.Masada, Modeling and Control of Power Plant Boiler-TurbineGenerator Systems, Sc.D.Thesis,MIT,Dept.of Mechanical Engineering, Nov. 1979.

[6] Compact Simulator Manual -Ormond Beach Unit2 Supercritical boiler/turbine-, Electric Power Research Institute and TRAX Corporation, 1993.

[7] J. P. LaSalle, The Stability of Dynamical Systems. CBMS-NSF 25, SIAM. Hamilton Press, Berlin, NJ, 1976. 